\title{
Redes sociais e bibliometria sobre a utilização de VANTs no mapeamento do risco hidrológico em áreas urbanas
}

\author{
Social networks and bibliometrics on the use of UAVs in hydrological risk mapping in urban areas \\ Redes sociales y bibliometría sobre el uso de UAV en el mapeo de riesgos hidrológicos en áreas
} urbanas

Recebido: 20/10/2021 | Revisado: 29/10/2021 | Aceito: 01/11/2021 | Publicado: 02/11/2021

Andrey Gaspar Sorrilha Rodrigues
ORCID: https://orcid.org/0000-0003-0149-1596
Universidade Federal de Mato Grosso do Sul, Brasil
E-mail: andreygaspar.agsr@ gmail.com
Alesson Pires Maciel Guirra
ORCID: https://orcid.org/0000-0003-4201-3574
E-mail: engeoguirra@ gmail.com
Daniella Nunes Silveira
Universidade Federal dos Vales do Jequitinhonha Mucuri, Brasil
ORCID: https://orcid.org/0000-0003-1987-3912
E-mail: daniella.n.silveira@ @mail.com
Amanda Letícia Abegg da Silveira
ORCID: https://orcid.org/0000-0001-9063-8793
Universidade Federal de Mato Grosso do Sul, Brasil
E-mail: abegg.geo@gmail.com
Jéssica Rabito Chaves
Ministério Público do Estado de Mato Grosso do Sul, Brasil
ORCID: https://orcid.org/0000-0003-2763-3361
Universidade Federal de Mato Grosso do Sul, Brasil
E-mail: je.rabitochaves@ gmail.com
Luiz Claudio Galvão do Valle Junior
ORCID: https://orcid.org/0000-0001-5708-4370
Universidade Federal de Mato Grosso do Sul, Brasil
E-mail: luizvallejr@ gmail.com
Antonio Conceição Paranhos Filho
ORCID: https://orcid.org/0000-0002-9838-5337
Universidade Federal de Mato Grosso do Sul, Brasil
E-mail: antonio.paranhos@ ufms.br
Roberto Macedo Gamarra
ORCID: https://orcid.org/0000-0002-0049-0009
Eniversidade Federal de Mato Grosso do Sul, Brasil
E-mail: roberto.gamarra@ ufms.br

\section{Resumo}

Este estudo visa realizar pesquisas na base de dados Scopus e Web of Science sob o intuito de procurar trabalhos relativos ao mapeamento hídrico com o enfoque em áreas de risco utilizando VANTs. O software VOSViewer, gera infográficos fundamentados no sistema de nós exibindo os principais termos da pesquisa, autores, elementos recorrentes e palavras-chave. Categorizando os trabalhos, de forma quantitativa, em autores colaboradores, abordagem referente ao tema, tipos de publicações, principais revistas, índices, principais países, redes de colaboração e palavras-chaves, indicou os principais trabalhos pertinentes ao tema, em que, esses foram discutidos para a verificação de como o tema é abordado. 91,15\% dos trabalhos estão escritos em inglês, sendo os EUA o segundo país que mais publica sobre, na retaguarda da China. Os principais termos concatenados a pesquisa são UAV - Unmanned Aerial Vehicles, Photogrammetry e DEM - Digital elevation Model, que fazem alusão ao uso de VANTs para obtenção do MDE e MDS pelo método fotogramétrico. Dentre os trabalhos, 10 apresentaram material metodológico e resultados promissores na utilização de VANT' para mapeamento de risco hidrológico, com destaque aos trabalhos de Mazzoleni, Muthusamy Annis, Luppichini, que apresentam softwares de processamento de imagem (Agisoft PhotoScan) e de simulações hidrodinâmicas de águas pluviais e fluviais (FLO-2D e HEC-RAS). Logo, esse estudo viabilizou reconhecer condutas metodológicas, tendências, dificuldades e as vantagens da ferramenta VANT, na aplicação técnica, como alta precisão, custo relativamente baixo e alcance em áreas de difícil acesso.

Palavras-chave: Drones, Modelos Digitais de Elevação (MDE); Modelos Digitais de Superfície (MDS); Fotogrametria; VOSViewer. 


\begin{abstract}
This study aims to carry out research in the Scopus and Web of Science databases in order to search for works related to water mapping with a focus on risk areas using UAVs. The VOSViewer software generates infographics based on the node system displaying the main search terms, authors, recurring elements and keywords. Quantitatively categorizing the works into collaborating authors, approach to the topic, types of publications, main journals, indexes, main countries, collaboration networks and keywords, he indicated the main works pertinent to the topic, in which these were discussed to verify how the topic is approached. $91.15 \%$ of the works are written in English, with the US being the second country that publishes the most about, in the rear of China. The main terms concatenated in the research are UAV - Unmanned Aerial Vehicles, Photogrammetry and DEM - Digital Elevation Model, which allude to the use of UAVs to obtain the MDE and MDS by the photogrammetric method. Among the works, 10 presented methodological material and promising results in the use of UAV' for hydrological risk mapping, with emphasis on the work of Mazzoleni, Muthusamy Annis, Luppichini, who present image processing software (Agisoft PhotoScan) and hydrodynamic simulations of water pluvial and fluvial (FLO-2D and HEC-RAS). Therefore, this study made it possible to recognize methodological approaches, trends, difficulties and the advantages of the UAV tool in technical application, such as high precision, relatively low cost and reach in areas of difficult access.
\end{abstract}

Keywords: Drones, Digital Elevation Models (DEM); Digital Surface Models (MDS); Photogrammetry; VOSViewer.

\title{
Resumen
}

Este estudio tiene como objetivo realizar una investigación en la base de datos Scopus y Web of Science con el fin de buscar trabajos relacionados con el mapeo del agua con un enfoque en áreas de riesgo utilizando UAV. El software VOSViewer genera infografías basadas en el sistema de nodos que muestran los principales términos de búsqueda, autores, elementos recurrentes y palabras clave. Categorizando cuantitativamente los trabajos en autores colaboradores, abordaje del tema, tipos de publicaciones, principales revistas, índices, principales países, redes de colaboración y palabras clave, señaló los principales trabajos pertinentes al tema, en los cuales se discutieron para una verificación de cómo se aborda el tema. El 91,15\% de las obras están escritas en inglés, siendo Estados Unidos el segundo país que más publica al respecto, en la retaguardia de China. Los principales términos vinculados a la investigación son UAV - Vehículos aéreos no tripulados, Fotogrametría y DEM - Modelo de elevación digital, que aluden al uso de UAV para obtener MDE y MDS por el método fotogramétrico. Entre los trabajos, 10 presentaron material metodológico y resultados prometedores en el uso de UAV' para el mapeo de riesgos hidrológicos, con énfasis en el trabajo de Mazzoleni, Muthusamy Annis, Luppichini, quienes presentan software de procesamiento de imágenes (Agisoft PhotoScan) y simulaciones de agua de lluvia y agua de río. (FLO-2D y HEC-RAS). Por lo tanto, este estudio permitió reconocer enfoques metodológicos, tendencias, dificultades y las ventajas de la herramienta UAV en la aplicación técnica, como alta precisión, costo relativamente bajo y alcance en áreas de difícil acceso.

Palabras clave: Drones, Modelos Digitales de Elevación (DEM); Modelos Digitales de Superficie (MDS); Fotogrametría; VOSViewer.

\section{Introdução}

O aumento da acessibilidade dos Veículos Aéreos Não Tripulados (VANTs), em especial os drones, instiga a utilização da ferramenta para diagnósticos e prognósticos com distintos respaldos técnicos em diversas áreas, sobretudo no setor ambiental, agrícola, de mineração, gestão e planejamento de áreas urbanas. Essas práticas fomentam o interesse de comercialização e a aquisição de novos dispositivos aéreos e sensores imageadores por empresas e instituições de ensino como Universidades e Institutos. As geotecnologias dos VANTs já existem a mais de 70 anos (Hardgrave, 2011), porém só recentemente o uso comercial e civil foi viabilizado (De Brum et al., 2019). Neste cenário, há um montante de informação que ainda está em desenvolvimento e a literatura científica sobre o tema abrange diversas formas de uso e aplicação destas ferramentas, dado ao seu caráter generalista de utilização.

A ANAC - Agência Nacional de Aviação Civil (2018) reconhece VANT como uma aeronave com alto grau de automatismo, não tripulada, remotamente pilotada e destinada a um grupo de operação, o não recreativo. Dentre as aeronaves que operam sem vínculo de piloto embarcado, os drones, têm sua representatividade como aeronaves que não possuem asas fixas e sim hélices rotativas, que conforme Paranhos Filho et al. (2016) são denominados de multirrotores.

Um dos possíveis produtos gerados por essas tecnologias são os Modelos Digitais de Elevação (MDE) e de Superfície (MDS), frutos dos processos da fotogrametria, que é a captação de imagens para a aplicação do efeito estereoscópico (Redweik, 2007). Permitindo a geração de modelos digitais de superfície com alto nível de detalhamento (Carrivick et al., 
2016).

Um MDE ou MDS traduz-se em uma representação matemática de uma matriz digital com atribuições de coordenadas a cada pixel, com valores aos eixos x, y e z, ou seja, são registrados valores de posicionamento e elevação, concebendo tridimensionalidade as manifestações geomorfométricas de uma superfície (Valeriano \& Rossetti, 2012). Existe a possibilidade de extrair diversas informações morfométricas de um espaço, através da análise de características da superfície ou terreno, como declividade, orientação de linhas de fluxo, direção de acúmulo, estrutura das curvas de nível, as quais quando associadas a parâmetros hídricos viabilizam a determinação de áreas potencialmente inundáveis, alagáveis e com riscos hidrológicos.

Riscos hidrológicos providos pelas áreas urbanas são deflagrados pelo ciclo periódico do curso natural da água, que devido às interações humanas com o ambiente podem ocasionar complicações socioambientais e socioeconômicas (Guerra \& Zacharias, 2016). Riscos de enchentes, alagamentos, enxurradas, inundações, deslizamento de terra e queda de barreiras são ameaças hidrológicas que oneram as pessoas de uma cidade (Rio Grande do Sul, 2016), provocando problemas de ordem econômica, social e ambiental. Portanto, ameaçar a vida e a qualidade de vida das pessoas, os patrimônios públicos e o desenvolvimento sustentável urbano vão contra o Estatuto da Cidade (Lei 10.257/2001) (Estatuto da Cidade, 2001), que prevê em lei o progresso urbanístico e as competências públicas provedoras.

Estudos que indagam discussões de mapeamento de risco e o ordenamento territorial são fundamentais para a preservação das cidades, tendo como função a contribuição em debates públicos e a formatação de políticas urbanas sustentáveis.

Como qualquer ferramenta, a usabilidade só se torna eficiente quando utilizada adequadamente e aliada a um método cientificamente consistente. Portanto, torna-se imprescindível analisar o conteúdo bibliográfico e as informações que fomentem direções que possam sustentar a pesquisa e a usabilidade. Garantindo credibilidade, rigor teórico e prático, compreendendo as lacunas significativas sobre este tema. A bibliometria é uma forma de incorporar o reconhecimento dos conteúdos publicados e designar o progresso de pesquisa, indicando principais tópicos, métodos, instituições de pesquisa, autores, citações e conteúdo de publicação. Foi realizada a análise da rede de pesquisa digital das bases Scopus e Web of Science $(W o S)$, fornecidos pelo acesso da UFMS - Universidade Federal de Mato Grosso do Sul, dentro de todo o período que incorpora o primeiro artigo publicado até as publicações mais atuais (2020). Dessa forma, esse estudo tem como objetivo assimilar as tendências de pesquisa em geotecnologias aplicadas com utilização de VANTs para mapeamento do risco hidrológico.

\section{Materiais e Métodos}

A abordagem da pesquisa teve como intenção documentar a produção técnica científica existente relacionada à utilização de VANTs para mapeamento de locais com risco hidrológico, publicados nas bases Scopus e Web of Science (WoS). Uma forma de analisar as informações do tema em ambas as bases foi realizar o mapeamento dos dados bibliométricos, identificando de forma quantitativa e qualitativa os principais autores colaboradores da pesquisa buscada, abordagem referente ao tema, tipos de publicações, principais revistas, índices (Índice-H e Fator de Impacto), principais países, redes de colaboração e palavras-chaves recorrentes.

\subsection{Termos de busca bibliográfica}

Foram feitas as pesquisas nas bases Scopus e Web of Science (WoS), as duas principais plataformas de busca por trabalhos científicos e acadêmicos de repercussão global, contendo publicações de diversos periódicos. Para a triagem das referências sobre o tema que relaciona o uso de VANTs dentro de áreas urbanas para mapeamento de locais com potencial risco hidrológico utilizamos os seguintes termos de caracteres em inglês, visando maior alcance nas buscas: 
- (uav* OR "unmanned aerial vehicle" OR "fixed wing" OR dron* OR rpa* OR "remotely piloted aircraft") AND (dem OR "digital elevation model" OR dsm OR "digital surface model") AND ( risk OR *flood* OR *flow* OR water*or inund*) NOT (vulcan* OR glaci* OR erosi* OR earthquake)

No filtro para a pesquisa foram examinados e ponderados exclusivamente artigos e artigos de revisão em pares, pois fornecem um maior volume informação que em outros tipos de trabalhos, como resumos ou resumos expandidos apresentados em conferências e simpósios. Dentre vários testes de termos de pesquisa, foram analisados quais responderiam melhor ao conteúdo procurado para a análise bibliométrica.

Foram realizados vários testes de termos de busca, alguns com a inserção de caracteres englobando expressões comuns na área do conhecimento em que a utilização de VANTs está inserida no geoprocessamento, como GIS, Geoprossesing ou/e Remote Sensing e a resposta na questão volumétrica de periódicos foi maior, porém disperso ao tema procurado. Os testes colaboraram na descoberta de quais termos usualmente eram associados aos trabalhos com VANTs e não tinham relação nenhuma com risco hidrológico, consequentemente, resultou na inserção dos seguintes caracteres, "NOT (vulcan* OR glaci* OR erosi* OR earthquake)", em que o NOT indica que os subsequentes termos não estarão contidos nos resultados da pesquisa.

\subsection{Mapeamento quantitativo e qualitativo}

As interfaces de mapeamento de conteúdo bibliográfico expõem expressões concisas e um bom efeito de visualização de dados. Desse modo, permitem tratar um banco de informações com uma grande quantidade e variedade de temas e interpor conexões e assimilações diversificadas, possibilitando uma cognição satisfatória da pesquisa (Moreira et al., 2020). Já existe disponível uma série de interfaces visuais de infográfico de informações digitais devido a popularização e a acessibilidade dessas ferramentas, tendo como exemplos o Pajek (Batagelj \& Mrvar, 2004), SciMAT (Cobo et al., 2012) e o VOSViewer (Van Eck \& Waltman, 2010), sendo este último, o programa utilizado para este estudo.

O VOSViewer demonstra graficamente e visualmente as informações de uma maneira fundamentada no sistema de nós, que consiste na identificação temática de cada nó e o distanciamento e a conexão, como o balizador de relação. A distância está associada proporcionalmente ao relacionamento, portanto, quanto maior for à distância entre os nós, menor é a relação, e vice-versa (Van Eck \& Waltman, 2010).

Com o material e informações literárias exportadas das bases Scopus e WoS, o conteúdo foi categorizado, organizado e descartado as duplicadas, pois por se tratarem de bases distintas é comum o mesmo artigo exportado estar em ambas as bases. Outra propriedade analisada é a disparidade das bases, pois quando importado os conteúdos a serem analisados, os layouts de exportação são completamente diferentes, necessitando da padronização do layout de informação para a inserção ao VOSViewer. Com o arranjo das redes e conexões entre os diversos trabalhos e autores, foi possível discutir como a pesquisa é abordada.

\section{Resultados e Discussão}

\subsection{Análise geral do termo de busca}

O total de trabalhos encontrados com os termos de pesquisa escolhidos, até o dia 28 de abril de 2020, foram 180 e 177 artigos, respectivamente nas Bases Scopus e WoS, totalizando 357 trabalhos. Dentre todos os trabalhos encontrados, 97 eram duplicatas. Assim, 260 é o valor do conjunto final de documentos.

Entre os trabalhos selecionados na busca, constatou-se que $91,15 \%$ dos trabalhos estão escritos na língua inglesa, seguido pelo chinês $(5,77 \%)$, coreano $(1,15 \%)$, alemão $(0,77)$, espanhol $(0,38 \%)$, francês $(0,38 \%)$ e português $(0,38 \%)$ (Figura 1). Nota-se também que $19,23 \%$ são de acesso aberto e $65,77 \%$ não possuem informação de acesso (Figura 2) e o restante dos 
artigos está dividido em tipos de acesso classificado pela Clarivate Analytics, nas classes Gold, Bronze e Green (15\%). Essa classificação da WoS, indica a base e o acesso dos trabalhos publicados. A classificação Gold refere-se a publicações em revistas listadas em bases de acesso aberto, a Bronze são publicações não listadas em bases de acesso aberto, mas podendo ser encontradas para a leitura no próprio site da editora e pôr fim, a Green, caracterizada por hospedagem institucional de trabalhos científicos, sem supervisão editorial.

Figura 1. Trabalhos escritos na língua inglesa, chinesa $(3,31 \%)$, coreano $(1,66 \%)$ e espanhol $(0,55 \%)$.

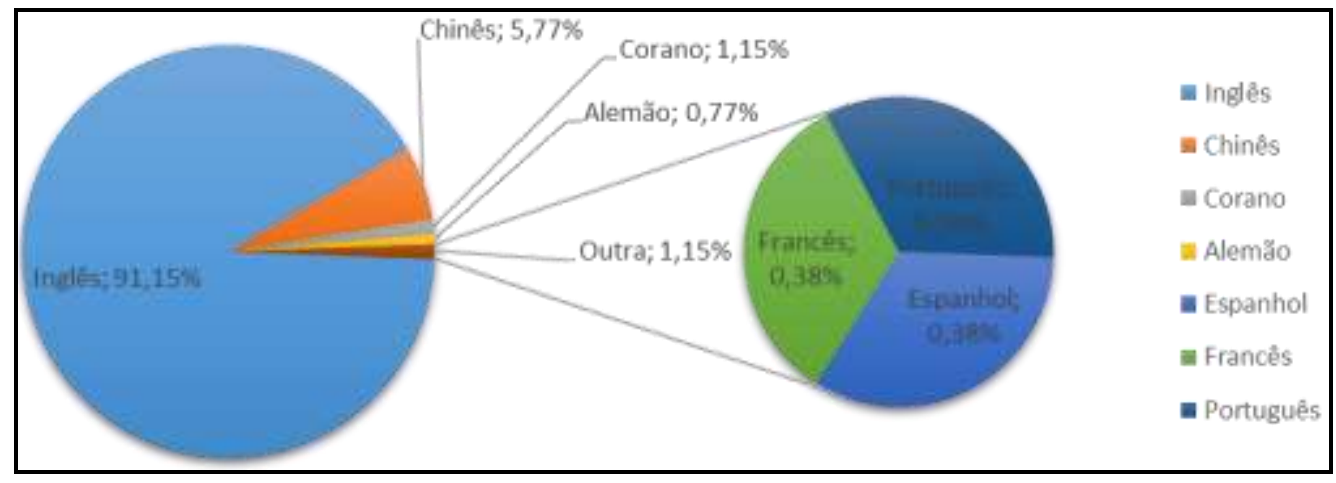

Fonte: Autores.

Figura 2. Tipo de acesso, sendo 19,23\% de acesso aberto, 65,77\% não identificados ou de acesso fechado e classes da Clarivate Analytics, Gold, Bronze e Green somando $15 \%$.

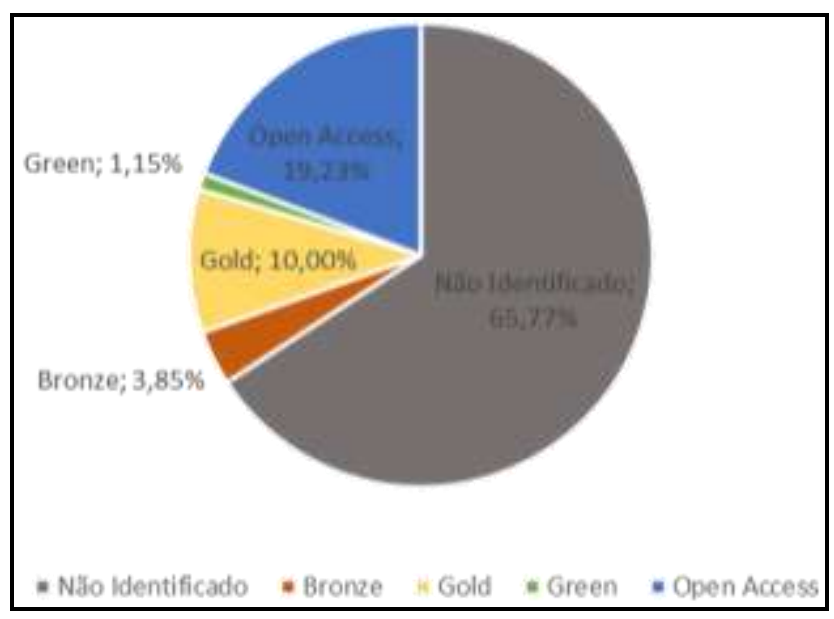

Fonte: Autores.

\subsection{Desenvolvimento dos trabalhos amostrados}

O primeiro texto encontrado com a pesquisa foi publicado em 2006, com o título de "Calibration of Small and LowCost UAV Video System for Real-Time Planimetric Mapping”, publicado pela editora IEEE - Institute of Electrical and Electronics Engineers. O estudo de Wu et al., (2006) aborda justamente uma maneira de melhorar os parâmetros de orientação da fotogrametria, com sugestão de uso para mapeamento de desastres naturais. No ano seguinte, encontra-se o segundo texto da busca, o "Digital elevation model based on aerial UAV image", em que Gu et al., (2007) explora a viabilidade dos sensores embarcados nos VANTs para geração de Modelos Digitais de Elevação e Superfície. Contudo, os pesquisadores dos dois trabalhos citados relataram dificuldades na produção de produtos fotogramétricos, oriundos de VANTs, com uma boa precisão. Os textos abordam técnicas que visam o melhoramento dos produtos gerados e também a correção dos problemas proporcionados pelo ambiente de voo e operacionalização. 
Talvez o problema dos dois autores esteja relacionado à carência de softwares com módulos matemáticos mais complexos que possam corrigir erros operacionais de voo como vento e trepidação. A falta de poder de processamento dos computadores no ano de 2006 e 2007 também é fator limitante da ferramenta, sendo o entrave enfrentado pelo uso de VANTs a mais de uma década atrás.

Dados significativos relacionados à utilização de VANTs, para mapeamento de áreas com potencial risco hidrológico, tiveram uma ascensão com a disponibilização de novos testes e ferramentas a partir de 2013 (Figura 3). O número de citações decresce gradualmente logo após o período de 2014, mas em contrapartida o volume de trabalhos continua crescente, isso provavelmente acontece devido à necessidade de exploração do potencial de uma tecnologia relativamente nova e ainda em desenvolvimento. Todavia, há também o fato de que uma grande quantidade de estudos ainda serem ainda pouco conhecidos e/ou reconhecidos no cenário científico.

Figura 3. O desempenho das publicações relacionadas à Utilização de VANTs em mapeamento de risco hidrológico obtidos da base de dados Scopus e WoS (2006-2020).

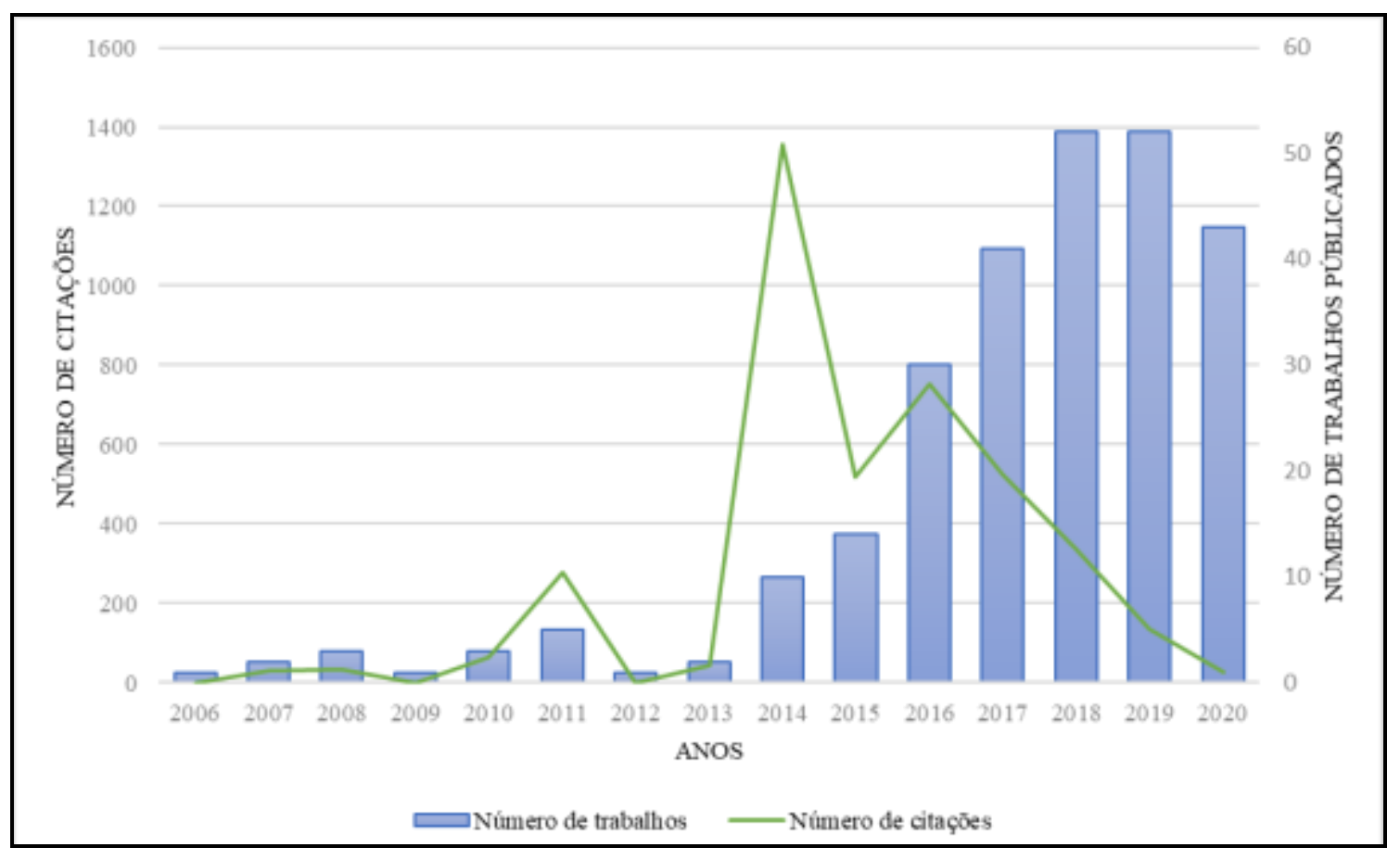

Fonte: Autores.

Existe um constante crescimento de produção de trabalhos a partir do ano de 2013 e 2014. Nota-se que a acessibilidade comercial da ferramenta, em especial os multirrotores (drones), começaram a se popularizar paralelamente ao desenvolvimento de novas abordagens de sensoriamento remoto vinculado à tecnologia (Mathusamy et al., 2019). Justificando a demanda de conhecimento em eventos, feiras, exposições, trabalhos científicos e correlatos.

No ano de 2013 foi lançada uma famosa série de drones no mercado, o Phantom, fabricado pela empresa chinesa DJIß (DJI, 2013), em que a sua popularidade dá-se ao valor, desenvolvimento, compatibilidade com softwares de operacionalização técnica e colaborativa com programas de processamento de imagens.

\subsection{Mapeamento do desempenho e desenvolvimento de palavras colaborativas a pesquisa (VOSViewer)}

O infográfico do conteúdo bibliográfico expõe as interações entre as palavras análogas, o progresso e o comportamento dos termos de pesquisa durante o tempo. Verifica-se que os 3 grandes grupos de palavras da pesquisa são $U A V$ 
- Unmanned Aerial Vehicles, Photogrammetry e DEM - Digital Elevation Model (Figura 4). Logo, incorpora-se a assimilação de que o DEM pode ser adquirido por um processo fotogramétrico de imagens captadas por um sensor embarcado a um veículo aéreo não tripulado.

Figura 4. Infográfico do mapeamento do conteúdo bibliográfico, relacionado às interações e desenvolvimento das palavraschaves junto a citações, bibliografia, autorias e coautorias.

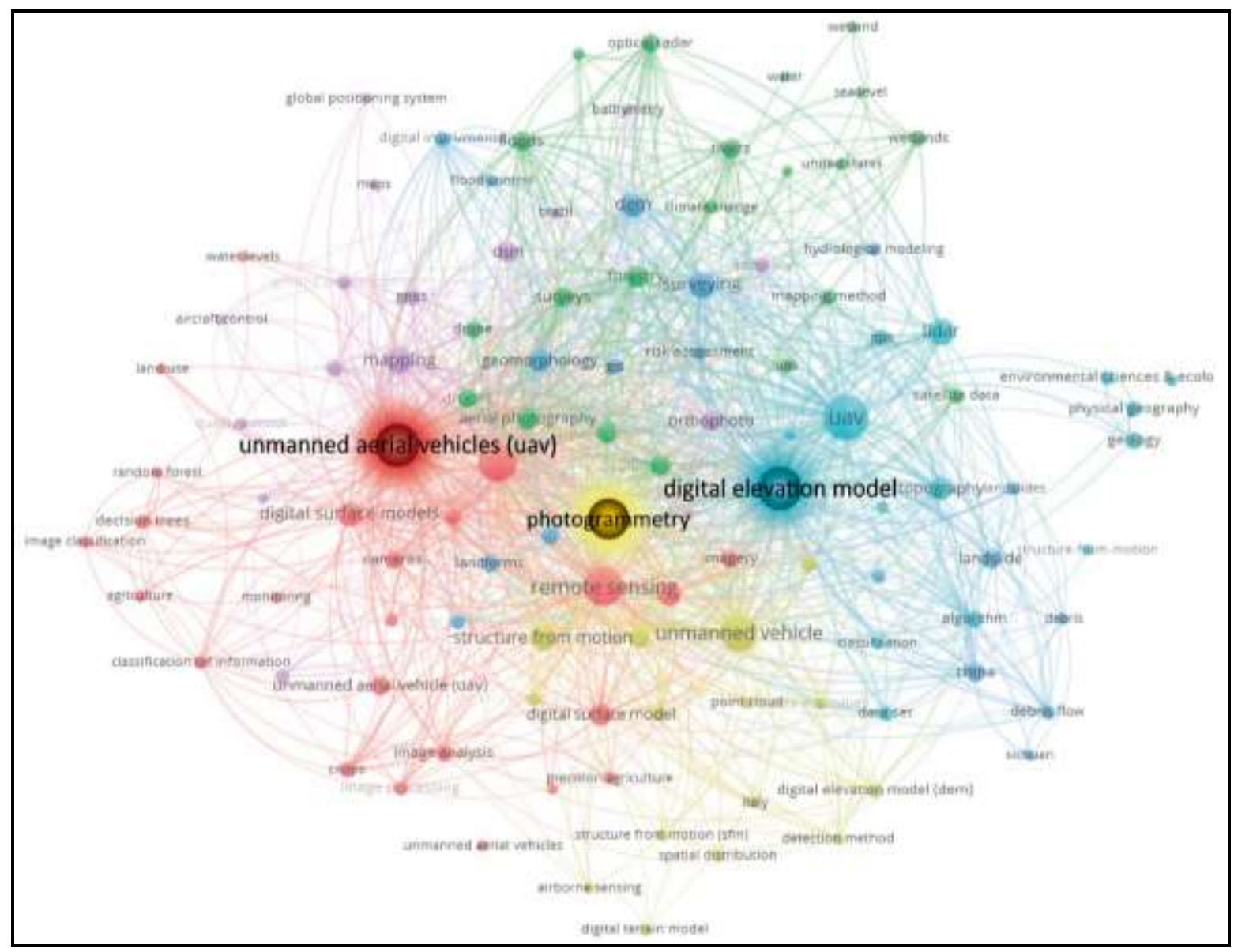

Fonte: Autores.

Os três termos destaques da Figura 4 são palavras com recorrências mais novas que Orthophoto, GIS, image processing ou até mesmo DEM e DSM (Figura 5). O fato dos termos UAVs, Photogrammetry e DEM serem mais citados recentemente decorre justamente da maior inserção na pesquisa e a "democratização" da tecnologia de VANTs dentro do sensoriamento remoto, mas existem ressalvas com o termo DEM.

A palavra DEM aparece como um termo comum dentro todo período de 2016 a 2019 (Figura 5) e isto deve-se às abordagens de processamento dos modelos digitais, que antes eram realizados por técnicas e fontes mais antigas e consolidadas, tais como: aeronaves embarcadas, satélites e outros sensores. O uso e o surgimento de novos testes, pesquisas e técnicas com produtos DEMs captados por sensores de $U A V s$, esclarece a distância temporal do mesmo termo em épocas diferentes. 
Figura 5. Infográfico do mapeando do conteúdo bibliográfico, com classificação de cores informando a data recorrente de cada termo, junto a interações e desenvolvimento das palavras-chaves junto a citações, bibliografia, autorias e coautorias.

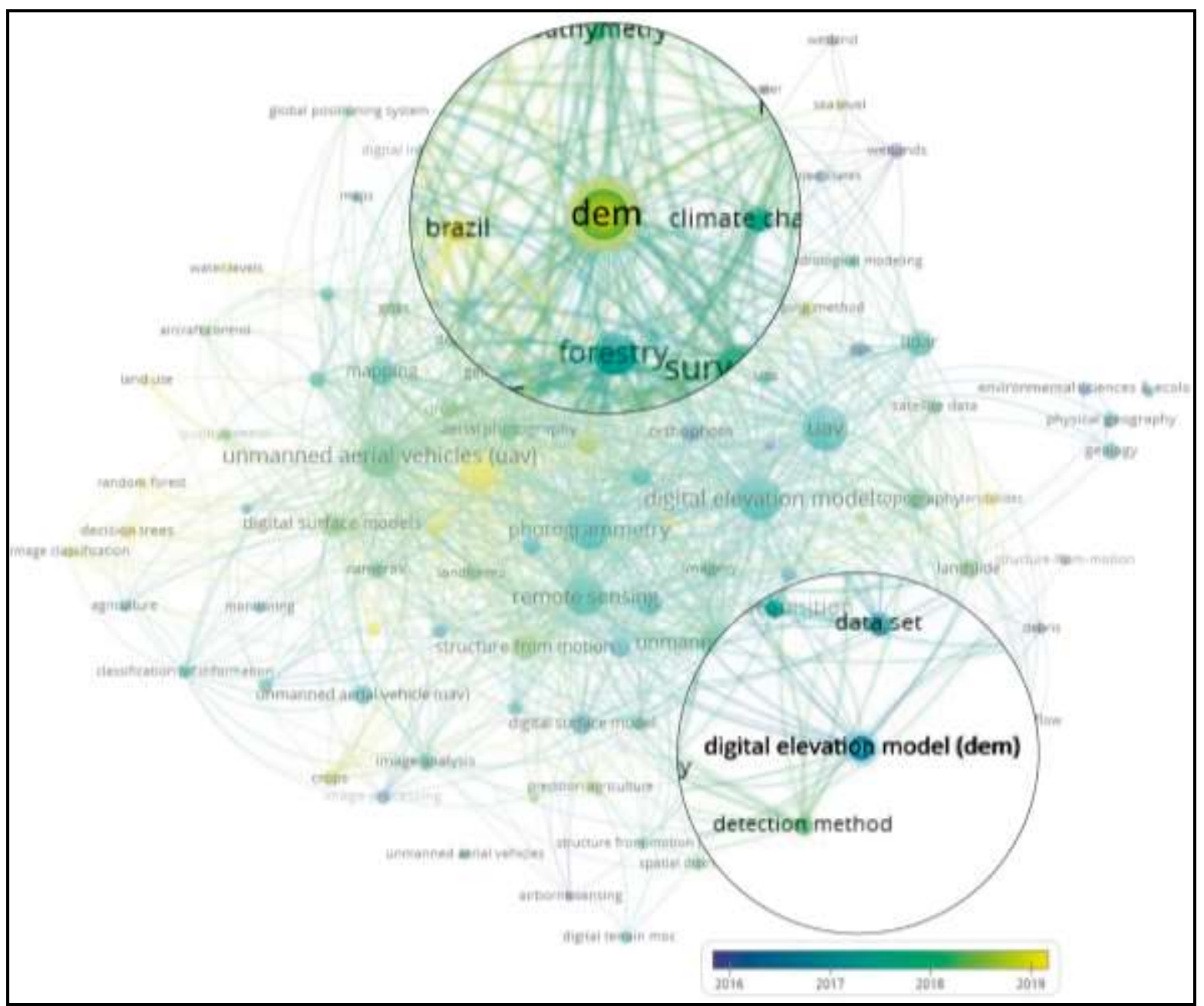

Fonte: Autores.

Com o infográfico reamostrado, identifica-se os 3 grandes grupos e as principais ramificações e interações. Termos recorrentes como drones, geomorphology, topography, flood, water levels e hydrological modeling (Figura 6) aparecem unidos, visto que, um dos principais fatores para a análise hidrológica, no que se refere a problemas de risco, é justamente a morfometria de um terreno e os critérios hidrológicos. A forma do ambiente é um dos parâmetros que moldam o curso de águas superficiais, sendo o detalhamento da inclinação em relação à superfície o critério métrico de um mapa topográfico (Paranhos Filho et al., 2020). 
Figura 6. Infográfico do mapeamento do conteúdo bibliográfico, com foco em UAV, Photogrammetry, Digital Elevation Model e suas segmentações, geomorphology, topography, flood control, relacionando interações e desenvolvimento das palavras-chaves junto a citações, bibliografia, autorias e coautorias.

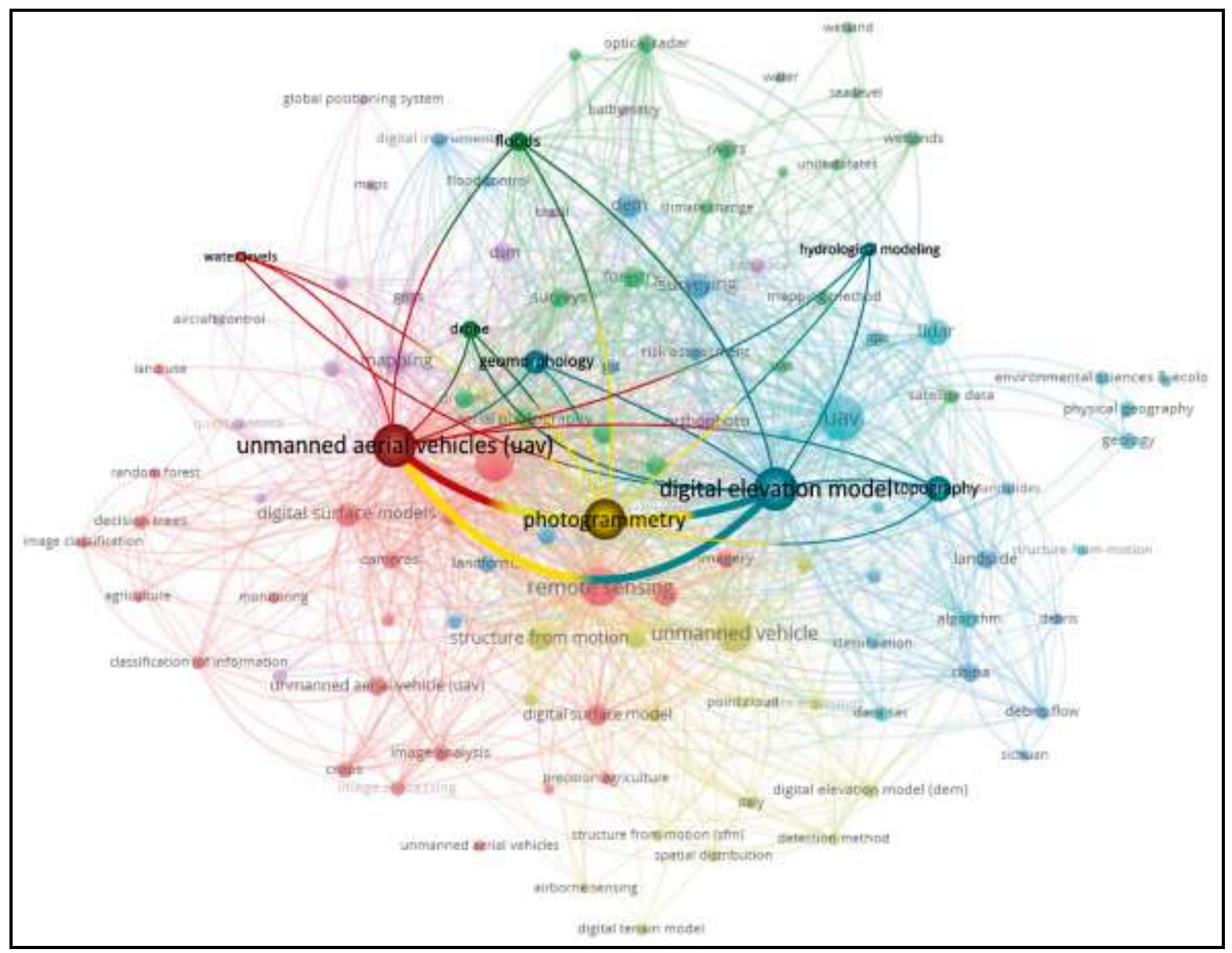

Fonte: Autores.

\subsection{Cooperação acadêmica mundial}

A identificação da afiliação de cada autor possibilitou o mapeamento da localização institucional científica por país (Figura 7). Entre as cooperações acadêmicas, destacaram-se a rede de pesquisadores chinesa e estadunidense, sendo os respectivos países os locais com maior quantidade de instituições que promovem o desenvolvimento e aplicação deste tema. O Brasil encontra-se em $6^{\circ}$ lugar, sendo que $96 \%$ dos trabalhos são produzidos por universidades, centros e institutos federais, demonstrando a importância da colaboração do ensino público superior no avanço tecnológico nacional, profissional e científico. 
Figura 7. Localização das instituições em que os colaboradores desenvolveram a pesquisa e a classificação da quantidade de trabalhos publicados por país.

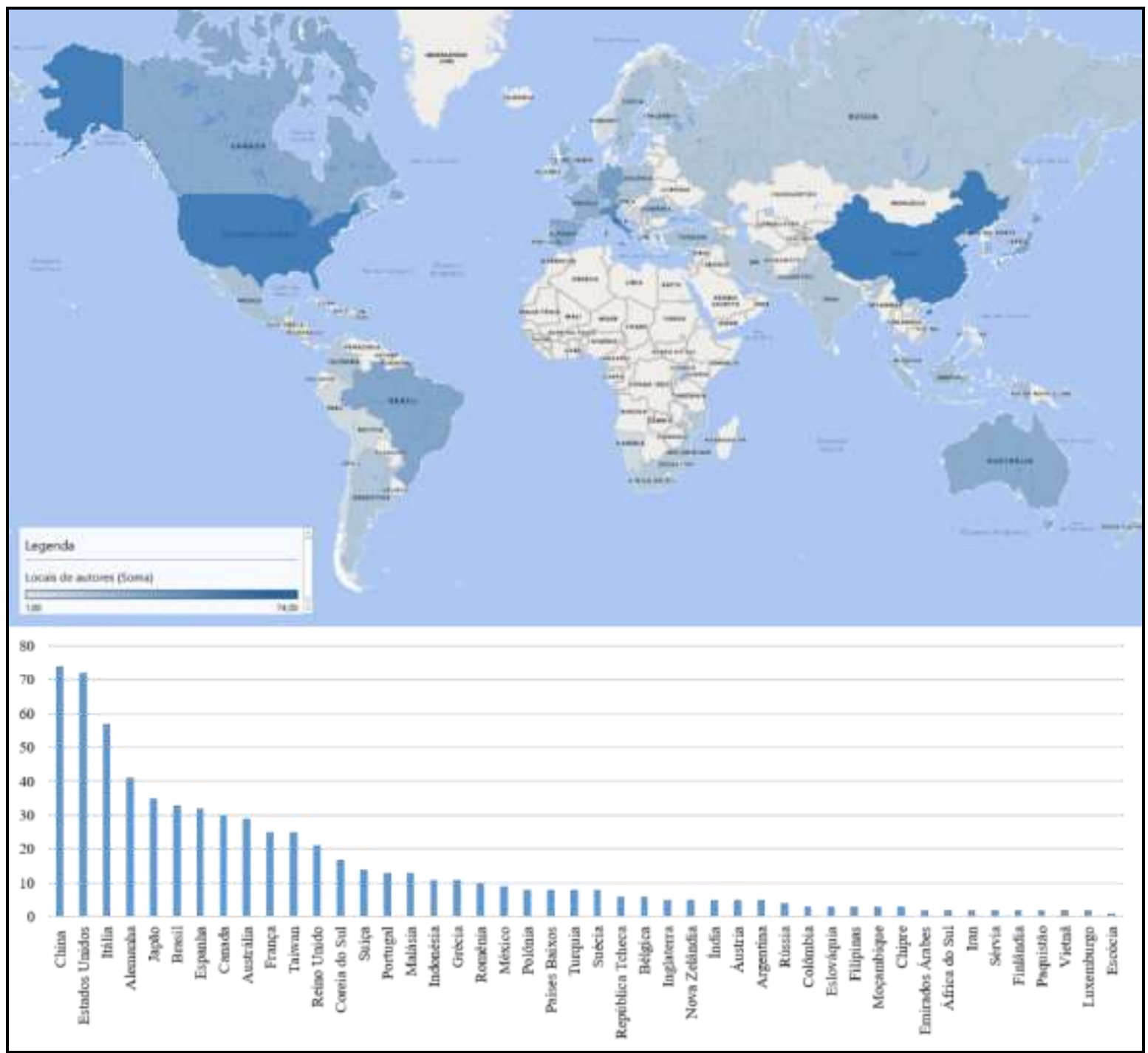

Fonte: Autores

Quantificando as cooperações científicas e correlacionando-as aos periódicos, destacam-se os periódicos "Remote Sensing”, “INT Arch Photogramm", "Sensors" $e$ "Geomorphology", pois possuem a maior quantidade de trabalhos publicados e estão entre as editoras com maior número de citações por trabalho (Tabela 1). A indicação dos locais onde foram encontrados muitos trabalhos com o reconhecimento da comunidade científica conduz a pesquisa e expõe periódicos propícios à publicação da mesma. Deste modo, é importante compreender e encontrar os principais meios divulgadores correlatos a geotecnologias aplicadas com utilização de VANTs para mapeamento do risco hidrológico. 
Tabela 1. Classificação de periódicos publicados por quantidade e número de citações e linhas destacadas em cinza indicam periódicos com maiores quantidades de trabalhos publicadas e editoras com maiores números de citações por trabalho.

\begin{tabular}{|c|c|c|c|c|c|c|}
\hline Periódico & Editoras & $\begin{array}{l}\text { País da } \\
\text { Revista }\end{array}$ & $\begin{array}{c}\mathbf{N}^{\circ} \text { de } \\
\text { Trabalhos } \\
\text { Publicados } \\
\end{array}$ & $\begin{array}{l}\sum \mathrm{N}^{0} \text { de } \\
\text { Citações }\end{array}$ & $\begin{array}{c}\text { Índice- } \\
\text { H }\end{array}$ & $\begin{array}{l}\text { Fator de } \\
\text { Impacto } \\
(2019-20) \\
\end{array}$ \\
\hline REMOTE SENSING & MDPI & Suíça & 16 & 378 & 99 & 4,59 \\
\hline $\begin{array}{c}\text { INT ARCH } \\
\text { PHOTOGRAMM }\end{array}$ & $\begin{array}{c}\text { COPERNICUS } \\
\text { GESELLSCHAFT } \\
\text { MBH }\end{array}$ & Alemanha & 12 & 113 & 64 & 0,93 \\
\hline PROC SPIE & $\begin{array}{c}\text { SPIE-INT SOC } \\
\text { OPTICAL } \\
\text { ENGINEERING }\end{array}$ & $\begin{array}{l}\text { Estados } \\
\text { Unidos }\end{array}$ & 8 & 55 & 162 & 1,28 \\
\hline SENSORS & MDPI & Suíça & 5 & 91 & 153 & 3,51 \\
\hline J COASTAL RES & $\begin{array}{c}\text { COASTAL } \\
\text { EDUCATION \& } \\
\text { RESEARCH } \\
\text { FOUNDATION }\end{array}$ & $\begin{array}{l}\text { Estados } \\
\text { Unidos }\end{array}$ & 4 & 4 & 84 & 1,04 \\
\hline $\begin{array}{c}\text { IOP C SER EARTH } \\
\text { ENV }\end{array}$ & $\begin{array}{l}\text { IOP PUBLISHING } \\
\text { LTD }\end{array}$ & Reino Unido & 4 & 4 & 18 & - \\
\hline GEOMORPHOLOGY & $\begin{array}{l}\text { ELSEVIER } \\
\text { SCIENCE BV }\end{array}$ & $\begin{array}{l}\text { Países } \\
\text { Baixos }\end{array}$ & 3 & 181 & 147 & 3,91 \\
\hline INT J REMOTE SENS & $\begin{array}{c}\text { TAYLOR \& } \\
\text { FRANCIS LTD }\end{array}$ & Reino Unido & 3 & 34 & 164 & 2,66 \\
\hline WATER-SUI & MDPI & Suíça & 3 & 23 & 42 & 2,59 \\
\hline $\begin{array}{c}\text { EARTH SURF PROC } \\
\text { LAND }\end{array}$ & WILEY & Reino Unido & 3 & 13 & 119 & 3,91 \\
\hline \multicolumn{7}{|c|}{ Classificação por Número de Citações } \\
\hline APPL. GEOMATICS & $\begin{array}{l}\text { SPRINGER } \\
\text { VERLAG }\end{array}$ & Alemanha & 1 & 669 & 18 & 1,2 \\
\hline REMOTE SENSING & MDPI & Suíça & 16 & 378 & 99 & 4,59 \\
\hline $\begin{array}{c}\text { ISPRS J } \\
\text { PHOTOGRAMM }\end{array}$ & $\begin{array}{l}\text { ELSEVIER } \\
\text { SCIENCE BV }\end{array}$ & $\begin{array}{l}\text { Países } \\
\text { Baixos }\end{array}$ & 2 & 220 & 126 & 8,19 \\
\hline GEOMORPHOLOGY & $\begin{array}{l}\text { ELSEVIER } \\
\text { SCIENCE BV }\end{array}$ & $\begin{array}{l}\text { Países } \\
\text { Baixos }\end{array}$ & 3 & 181 & 147 & 3,91 \\
\hline FRONT PLANT SCI & $\begin{array}{l}\text { FRONTIERS } \\
\text { MEDIA SA }\end{array}$ & Suíça & 2 & 142 & 101 & 4,3 \\
\hline $\begin{array}{c}\text { INT J APPL EARTH } \\
\text { OBS }\end{array}$ & ELSEVIER & $\begin{array}{l}\text { Países } \\
\text { Baixos }\end{array}$ & 1 & 142 & 86 & 5,34 \\
\hline J ARCHAEOL SCI & $\begin{array}{c}\text { ACADEMIC } \\
\text { PRESS LTD- } \\
\text { ELSEVIER } \\
\text { SCIENCE LTD }\end{array}$ & $\begin{array}{l}\text { Estados } \\
\text { Unidos }\end{array}$ & 2 & 125 & 118 & 3,17 \\
\hline $\begin{array}{c}\text { INT ARCH } \\
\text { PHOTOGRAMM }\end{array}$ & $\begin{array}{c}\text { COPERNICUS } \\
\text { GESELLSCHAFT } \\
\text { MBH }\end{array}$ & Alemanha & 12 & 113 & 64 & 0,93 \\
\hline PHOTOGRAMM REC & WILEY & Reino Unido & 2 & 105 & 48 & 1,92 \\
\hline SENSORS & MDPI & Suíça & 5 & 91 & 153 & 3,51 \\
\hline
\end{tabular}

Fonte: Autores.

É relevante destacar o periódico “APPL. GEOMATICS”, visto que dispõe de apenas um trabalho publicado com citações maiores que qualquer outro. O trabalho divulgado por essa revista foi o "UAV for $3 D$ mapping applications: $A$ review” dos autores Nex e Remondino (2014). Neste artigo é abordado um panorama geral sobre os VANTs, debatendo em um a diferenciação de categoria de aeronaves, tipos de processamento, regularização entre os países, planejamento de pontos de controle, planejamento de voo, plataformas de operacionalização, estudos de casos, aplicações e limitações enfrentadas com a tecnologia na época. 
É interessante observar as diferentes perspectivas de Nex e Remondino (2014), pois abordam outras aplicações, dificuldades, concepções, restrições e o desenvolvimento da ferramenta até a realidade de 2020, quando comparado a trabalhos mais recentes. $\mathrm{O}$ artigo debate as limitações referentes à autonomia de voo, problemas de estabilidade e a carência de computadores com processamento de dados mais rápido para aplicações que requerem alta precisão, no entanto, esses apontamentos já não são mais empecilhos de contenção dos VANTs após 6 anos de desenvolvimento científico. Contudo, as certificações de segurança, legislações e as solicitações de permissões de voo, citados pelos pesquisadores, ainda são um impedimento recorrente da ferramenta.

A utilização de novos sensores a bordo como térmicos, multiespectrais e dispositivo RTK embarcado para estudos futuros também é discutido no periódico. Essas implementações tecnológicas melhorariam a qualidade do posicionamento e informação, porém a precificação seria elevada, indo contra o intuito dos VANTs de uma combinação de solução de baixo custo. A respeito de aplicações, quando relacionado à análise hidrológica, os VANTs proporcionam atividades de monitoramento, planejamento, gerenciamento de impacto de águas e informações morfométricas da superfície de interesse, como estabilidade dos terrenos, declividades, cálculo de volumes, etc (Nex \& Remondino, 2014).

\subsection{Desenvolvimento de pesquisas para a "Utilização de VANTs em mapeamento de risco hidrológico"}

Com a resposta concebida pelos infográficos e a categorização dos periódicos com maior quantidade de trabalho e citação, revelaram os artigos compatíveis (Tabela 2) ao tema sobre a utilização de VANTs em mapeamento de risco hidrológico.

Tabela 2. Trabalhos que fazem relação à utilização de VANTs em mapeamento de risco hidrológico, com foco no gerenciamento de risco através de Modelos Digitais.

\begin{tabular}{lll}
\hline Nome do trabalho & Ano & Autores \\
\hline $\begin{array}{l}\text { UAV-DEMs for small-scale flood hazard mapping } \\
\text { Testing UAV-derived topography for hydraulic modelling in a tropical }\end{array}$ & 2020 & Annis et al. 2019 \\
environment & Mazzoleni et al. (2020) \\
$\begin{array}{l}\text { Influence of topographic resolution and accuracy on hydraulic channel flow } \\
\text { simulations: Case study of the Versilia River (Italy) }\end{array}$ & 2019 & Luppichini et al. (2019) \\
$\begin{array}{l}\text { A remote sensing based integrated approach to quantify the impact of fluvial } \\
\text { and pluvial flooding in an urban catchment }\end{array}$ & 2019 & Muthusamy et al. (2019) \\
$\begin{array}{l}\text { Analysis the Accuracy of Digital Elevation Model (DEM) for Flood Modelling } \\
\text { on Lowland Area }\end{array}$ & 2018 & Abidin et al. (2018) \\
$\begin{array}{l}\text { An investigation of Digital Elevation Model (DEM) structure influence on } \\
\text { flood modelling }\end{array}$ & 2018 & Sahid et al. (2018) \\
$\begin{array}{l}\text { Generation of high-resolution digital surface models for urban flood } \\
\text { modelling using UAV imagery }\end{array}$ & 2018 & Yalcin (2018) \\
$\begin{array}{l}\text { Improving a DSM Obtained by Unmanned Aerial Vehicles for Flood } \\
\text { Modelling }\end{array}$ & 2017 & Mourato et al. (2017) \\
$\begin{array}{l}\text { Assessing the quality of digital elevation models obtained from mini } \\
\text { unmanned aerial vehicles for overland flow modelling in urban areas }\end{array}$ & 2016 & Leitão et al. (2016) \\
$\begin{array}{l}\text { Flood risk management in remote and impoverished areas - a case study of } \\
\text { Onaville, Haiti }\end{array}$ & 2015 & Heimhuber et al. (2015) \\
\hline
\end{tabular}

\section{Fonte: Autores.}

A operação autônoma dos VANTs estende a utilização da ferramenta, visto que não há a necessidade de piloto embarcado, pois a aeronave é controlada remotamente (Leitão et al., 2016), logo, abrange inúmeras vantagens como custo, tempo para levantamento de informações e queda nos riscos operacionais (Mazzoleni et al., 2020). Os benefícios da tecnologia fomentaram sua popularização e consequentemente, os helicópteros de múltiplas lâminas habitualmente conhecidos com 
drones (Leitão et al., 2016; Muthusamy et al., 2019) o uso por civis, que dobrou entre os anos de 2008 a 2014 (Colomina \& Molina, 2014).

O $D E M$ é fruto de uma série de processamentos de imagens que podem ser adquiridas por meio de sensores embarcados por VANTs. O texto "Assessing the quality of digital elevation models obtained from mini unmanned aerial vehicles for overland flow modelling in urban areas" (Leitão et al., 2016) discute a importância da qualidade dos modelos quando associado a processo hidrológico e como realizar esse aprimoramento. Altitudes baixas, maior sobreposição de imagens, condições climáticas em que o sol não causaria desbalanço nos tons de branco (melhores imagens em dias nublados) e o uso das imagens como parâmetro de classificação do solo para processos superficiais, são tópicos significativos no aperfeiçoamento do DEM (Leitão et al., 2016).

Em "Generation of high-resolution digital surface models for urban flood modelling using uav imagery" (Yalcin, 2018) o autor estabelece que quando se faz necessário simular o comportamento hidrológico em superfícies urbanas complexas, modelos digitais com alta resolução se fazem imprescindíveis. Resultados da simulação sobre a extensão da inundação, profundidade da água e velocidades de fluxo em áreas urbanas são variáveis voláteis a resolução do DEM (Abdin et al., 2018; Leitão et al., 2016; Luppichini et al., 2019; Yalcin, 2018).

Dados topográficos de alta resolução são pré-requisitos para simular com precisão o movimento da água, sendo um dos fatores mais impactantes para a precisão dos modelos de risco hidrológico, porém fatores pluviométricos, rugosidade e cisalhamento também tem seu papel fundamental na combinação de características da superfície. Existem outros critérios relevantes na conduta hidrológica em áreas urbanas como rugosidade da cobertura do solo (Luppichini et al., 2019; Mourato et al., 2017). O volume de água que infiltra ou não, consequentemente afeta o acúmulo e velocidade, visto que problemas de alagamento, inundação ou enchentes são processos naturais, todavia, catalisados por intervenções antrópicas.

\subsection{Resumo das Abordagens metodológicas na utilização de VANTs em mapeamento de risco hidrológico em áreas urbanas}

O entendimento do tema por revisões de trabalhos esclarece diversas dúvidas, mas essencialmente a de como realizar os processos operativos. Mapear as áreas de riscos requer um método científico consistente e ajustado ao local pesquisado, aos recursos de pesquisas e aos dados coletados no local. Seguindo essas especificações os trabalhos foram sintetizados em:

- “Generation of high-resolution digital surface models for urban flood modelling using uav imagery”,Yalcin (2018) esclarece a maneira de executar o mapeamento com drones e indaga a necessidade de precisão na geração dos DEM.

- "Assessing the quality of digital elevation models obtained from mini unmanned aerial vehicles for overland flow modelling in urban áreas", Leitão et al., (2016) faz constatações relacionadas aos modos operantes com os VANTs como altitudes de voos, sobreposição de imagens, condições do clima, inclinação do sensor embarcado e o uso fundamental dos pontos de controle.

- "Analysis the Accuracy of Digital Elevation Model (DEM) for Flood Modelling on Lowland Area”, Abdin et al., (2018) demonstra um dos softwares de processamento de imagens utilizado para a geração de modelos digitais de superfície e elevação, o Agisoft PhotoScan (Agisoft, 2018) Também certifica a diferenciação entre as qualidades de processamento opcionais do programa (baixo, médio e alto).

- "Flood Risk Management in Remote and Impoverished Areas - A Case Study of Onaville, Haiti", Heimhuber et al., (2015) discute parâmetros hidráulicos de um sistema hidrológico (curvas IDF - Intensidade e Duração e frequência, índices de chuva e rugosidade) e como incorporá-lo em um mapa base para 
gerenciamento de risco hídrico. Apresenta também as extensões gratuitas para o software ArcGIS (2012), o HEC-GeoHAS (USACE, 2010)_e o HEC-GeoHMS (USACE, 2013).

- "Testing UAV-derived topography for hydraulic modelling in a tropical environment", Mazzoleni et al., (2022) discorre acerca de novos sensores embarcados nos VANTs, como o LiDAR, RTK e entre outros. Constata-se que essas novas abordagens auxiliam em muito a precisão e no aprimoramento da tecnologia, em contrapartida, o custo dos sensores e a adaptação aos VANTs, até então (2020), são elevadas, indo em sentido oposto à proposta de uma ferramenta acessível.

- "Improving a DSM Obtained by Unmanned Aerial Vehicles for Flood Modelling”, Mourato et al., (2017) faz abordagens conceituais demonstrando como aperfeiçoar a precisão dos modelos digitais e descreve a importância da composição detalhada da superfície no processo hidrológico urbano.

- "A Remote Sensing Based Integrated Approach to Quantify the Impact of Fluvial and Pluvial Flooding in an Urban Catchment", Muthusamy et al., (2019) explora a importância dos modelos 2D no gerenciamento de risco de inundação baseado em DEM e que dados coletados em campo validam as previsões das modelagens hídricas e calibram o modelo.

- "UAV-DEMs for Small-Scale Flood Hazard Mapping", Annis et al., (2020) apresenta a "Abordagem Baseada em Eventos para Bacias Pequenas e Não Medidas (EBA4SUB)", fornecendo uma estimativa aplicada a uma simulação em cenário de chuva real. Também analisa a necessidade da precisão no modelo digital de elevação e divulga o software para a execução da simulação, o FLO-2D (2009) que funciona com uma extensão gratuita para o QGIS (2020).

- "Influence of Topographic Resolution and Accuracy on Hydraulic Channel Flow Simulations: Case Study of the Versilia River (Italy)”, Luppichini et al., (2019) faz aplicação em um estudo de caso e apresenta o comportamento hidrológico urbano simulado em diferentes resoluções, aplicando o modelo matemático do FLO-2D (2009).

\section{Conclusão}

O conteúdo bibliográfico devidamente catalogado no VOSViewer pelo critério de cadeia de caracteres, possibilitou a análise de inter-relações entre tópicos e temáticas como: autores, coautorias, citações, palavras-chaves e periódicos relacionados à utilização de VANTs para mapeamento de locais com risco hidrológico. Logo, viabilizou identificar, como os autores abordam de forma metodológica a utilização de VANTs em mapeamento de risco hidrológico e as tendências e dificuldades enfrentadas.

Existem vantagens do sensoriamento remoto baseado na utilização de VANTS, em especial os drones, como uma redução drástica nos riscos operacionais, custos e tempo de execução e ainda fornecem MDS e MDE precisos e de altíssima resolução (Annis et al., 2020; Mozzoleni et al., 2020). Os trabalhos demonstraram que um modelo digital de elevação e superfície de alta resolução é necessário para simular com precisão os efeitos de possíveis riscos hidrológicos, como alagamento, inundação e enxurradas (Yalcin et al., 2018; Leitão et al., 2016; Mazzonelli et al., 2020; Luppichini et al., 2019), corrigindo sub e/ou superestimativas oriundas de imagens de radar convencionais, nas quais predominam resoluções espaciais de maior metragem.

Recomendamos trabalhos futuros de cienciometria e/ou bibliometria para verificar a utilização de VANTs em mapeamento de risco hidrológico no Brasil, buscando ranquear as principais técnicas operacionais e se os planos diretores municipais vêm aplicando estas ferramentas na gestão do uso do solo, sobretudo no que diz respeito ao controle integrado das drenagens. 


\section{Agradecimentos}

Os autores agradecem ao PGRN/UFMS (Programa de Pós-Graduação em Recursos Naturais), da Universidade Federal de Mato Grosso do Sul - UFMS. O presente trabalho foi realizado com apoio da Coordenação de Aperfeiçoamento de Pessoal de Nível Superior - Brasil (CAPES) - Código de Financiamento 001. Agradecemos ainda a CAPES pelo acesso ao Portal de Periódicos. Ao CNPq (Conselho Nacional de Desenvolvimento Científico e Tecnológico) pela bolsa de produtividade em pesquisa de A. C. Paranhos Filho (CNPq Processo 305013/2018-1). O presente trabalho foi realizado com apoio da Fundação Universidade Federal de Mato Grosso do Sul - UFMS/MEC -Brasil.

\section{Referências}

Abidin, K. H. Z. K. Z. Razi, M. A. M. \& Bukari, S. M. (2018). Analysis the Accuracy of Digital Elevation Model (DEM) for Flood Modelling on Lowland Area. In IOP Conference Series: Earth and Environmental Science, IOP Publishing. 140, 1, 012014. 10.1088/1755-1315/140/1/012014

About ArcGIS. Mapping \& Analytics Software and Services. 10.1 Version, 2012. https://www.esri.com/en-us/arcgis/about-arcgis/overview.

Agência Nacional de Aviação Civil ANAC Regulamento Brasileiro (2018). Especial-RBAC-E n ${ }^{\circ}$ 94. Requisitos Gerais para Aeronaves Não Tripuladas de Uso Civil. Resolução, n. 419. <https://www.anac.gov.br/assuntos/legislacao/legislacao-1/rbha-e-rbac/rbac/rbac-e-94-emd-01/@@ displayfile/arquivo_norma/RBACE94EMD01.pdf>

Agisoft LLC Agisoft PhotoScan User Manual Professional Edition, Version 1.5, 2018. https://www.agisoft.com/pdf/photoscan-pro_1_4_en.pdf.

Annis, A.; Nardi, F.; Petroselli, A.; Apollonio, C.; Arcangeletti, E.; Tauro, F.; \& Grimaldi, S (2020). UAV-DEMs for small-scale flood hazard mapping. Water, 12(6), 1717. https://doi.org/10.3390/w12061717 Hardgrave, O. Pioneirismo com o Vant: Estados Unidos da América, 2005. http://www.ctie.monash.edu.au/hardgrave/

Batagelj, V.; \& Mrvar (2004). A Program for analysis and visualization of large networks. Ljubljana, Slovenia. http://vlado.fmf.uni1j.si/pub/networks/pajek/doc/pajekman.htm>

Carrivick, J. L. Smith, M. W. \& Quincey, D. J (2016). Structure from Motion in the Geosciences. John Wiley \& Sons. 1, 34. https://www.wiley.com/enus/Structure+from+Motion+in+the+Geosciences-p-9781118895849

Cobo, M. J. López-Herrera, A. G. Herrera-Viedma, E. \& Herrera, F. (2012). SciMAT: A new science mapping analysis software tool. Journal of the American Society for Information Science and Tecnology, 63, 8, 1609-1630. https://doi.org/10.1002/asi.22688

Colomina, I. \& Molina, P. (2014). Unmanned aerial systems for photogrammetry and remote sensing: A review. ISPRS Journal of Photogrammetry and Remote Sensing, 92, 79-97. https://doi.org/10.1016/j.isprsjprs.2014.02.013

De Brum, C. B. Mauricio, M. Da Silva, R. O. \& Bastos, Y. (2019). In: Uso dos drones nos procedimentos civis e criminais no Brasil: considerações sob a ótica dos direitos fundamentais. Prudkin G, Breuning, FM (Org). UFMS. https://repositorio.ufsm.br/bitstream/handle/1/18774/DR ONES\%20e\%20C IENCIA.pdf?sequence $=1 \&$ isAllowed $=\mathrm{y}$

DJI. Phantom User Manual, v1.1; DJI: Shenzhen, China, 2013. <https://www.dji.com/br/downloads/products/phantom>

Estatuto da Cidade (2001). Lei n. 10.257, de 10 de julho de 2001. Regulamenta os artigos, 182. http://www.planalto.g ov.br/ccivil_03/leis/leis_2001/110257.htm

FLO-2D Software FLO-2D Reference Manual; FLO-2D Software, Inc. P.O. AZ, USA, 2009. https://flo-2d.com/.

Gu, N. Feng, Z. K. \& Luo, X. (2007). Digital elevation model based on aerial UAV image. Beijing Linye Daxue Xuebao/Journal of Beijing Forestry University, 29, 152-155. http://caod.oriprobe.com/articles/25904714/Digital_elevation_model_based_on_aerial_UAV_image_htm

Guerra, F. C. \& Zacharias, A. A. (2016). Mapeamento das áreas de riscos hidrológicos e as políticas públicas de sustentabilidade: o caso de Ourinhos/SP. Revista Nacional de Gerenciamento de Cidades, 4(26), 223 - 243. https://doi.org/10.17271/2318847242620161345

Heimhuber, V. Hannemann, J. C. \& Rieger, W. (2015). Flood risk management in remote and impoverished areas - A case study of Onaville, Haiti. Water. 7(7), 3832-3860. https://doi.org/10.3390/w7073832

Leitão, J. P. Moy de Vitry, M. Scheidegger, A. \& Rieckermann, J. (2016). Assessing the quality of digital elevation models obtained from mini unmanned aerial vehicles for overland flow modelling in urban areas. Hydrology and Earth System Sciences, 20(4), 1637-1653. https://doi.org/10.5194/hess-20-16372016

Luppichini, M. Favalli, M. Isola, I. Nannipieri, L. Giannecchini, R. \& Bini, M. (2019). Influence of topographic resolution and accuracy on hydraulic channel flow simulations: case study of the Versilia River (Italy). Remote Sensing, 11(13), 1630. https://doi.org/10.3390/rs11131630

Mazzoleni, M.; Paron, P.; Reali, A.; Juizo, D.; Manane, J.; \& Brandimarte, L. Testing UAV-derived topography for hydraulic modelling in a tropical environment (2020), Natural Hazards, 103(1), 139-163. https://doi.org/10.1007/s11069-020-03963-4

Moreira, P. S. C. Guimarães, A. J. R. \& Tsunoda, D. F. (2020). Qual ferramenta bibliométrica escolher? Um estudo comparativo entre softwares. P2p $e$ Inovação, 6, 140-158. https://doi.org/10.21721/p2p.2020v6n2.p140-158 
Mourato, S. Fernandez, P. Pereira, L. \& Moreira, M. (2017). Improving a DSM obtained by unmanned aerial vehicles for flood modelling. In IOP Conference Series: Earth and Environmental Science, IOP Publishing, 95, 2, 022014. 10.1088/1755-1315/95/2/022014

Muthusamy, M. Rivas Casado, M. Salmoral, G. Irvine, T. \& Leinster, P. (2019). A remote sensing based integrated approach to quantify the impact of fluvial and pluvial flooding in an urban catchment. Remote Sensing. 11(5), 577. https://doi.org/10.3390/rs11050577

Nex, F. \& Remondino, F. (2014). UAV for 3D mapping applications: a review. Applied Geomatics. 6(1), 1-15. https://doi.org/10.1007/s12518-013-0120-x

Paranhos Filho, A. C. Mioto, C. L. Junior, J. M (2016). Geotecnologias em aplicações ambientais. Editora UFMS, 1, 6-15. https://editora.ufms.br/produto/geotecnologias-em-aplicacoes-ambientais/

Paranhos Filho, A. C. Mioto, C. L. Pessi, D. D. Gamarra, R. M.; Da Silva, N. M.; Ribeiro, V. O.; \& Chaves, J. R. (2020). Geotecnologias para aplicações ambientais. Ed. Uniedusul, 1, 224-235. https://www.uniedusul.com.br/wp-content/uploads/2021/01/GEOTECNOLOGIAS-PARA-APLICACOESAMBIENTAIS.pdf

QGIS API. 2020. QGIS API Documentation. https://qgis.org/api/3.4/

Redweik, P. (2007). Fotogrametria aérea. Faculdade de Ciências da Universidade de Lisboa, Lisboa, 1, 1-36. <https://www.researchgate.net/profile/PaulaRedweik/publication/268329721_FOTOGRAMETRIA_AEREA/links/564da1e608ae1ef9296aba8d/FOTOGRAMETRIA-AEREA.pdf>

Rio Grande do Sul, Brasil (2016). Ministério Público do Estado do Rio Grande do Sul. Promotoria de Justiça. Áreas de risco ocupações em planícies de inundação. 22. https://www.mprs.mp.br/noticias/ambiente/41036/

Sahid, N. A. W. \& Hadi, M. P. (2018). An investigation of Digital Elevation Model (DEM) structure influence on flood modelling. In IOP Conference Series: Earth and Environmental Science, IOP Publishing, 148, 1, 012001. 10.1088/1755-1315/148/1/012001

USACE. User's Manual HEC-GeoHAS 9.3; Hydrologic Engineering Center-Geospatial Hydrologic Modelling System; US Army Corps of Engineers: Washington, DC, USA, 2010. https://www.hec.usace.army.mil/software/hec-georas/downloads.aspx

USACE. User's Manual HEC-GeoHMS 10.1; Hydrologic Engineering Center-Geospatial Hydrologic Modelling System; US Army Corps of Engineers: Washington, DC, USA, 2013. https://www.hec.usace.army.mil/software/hec-geohms/downloads.aspx

Valeriano, M. M. \& Rossetti, D. F. (2012). Topodata: Brazilian full coverage refinement of SRTM data. Applied Geography, Elsevier, 32, 300-309. https://doi.org/10.1016/j.apgeog.2011.05.004

Van Eck, N. J. \& Waltman, L. (2010). Software survey: VOSViewer, a computer program for bibliometric mapping. Scientometrics. 84, 523-538. https://doi.org/10.1007/s11192-009-0146-3

Wu, J. Zhou, G. \& Li, Q. (2006). Calibration of small and low-cost UAV video system for real-time planimetric mapping. In 2006 IEEE International Symposium on Geoscience and Remote Sensing, IEEE. 10.1109/IGARSS.2006.535

Yalcin, E. M. R. A. H. (2018). Generation of high-resolution digital surface models for urban flood modelling using UAV imagery. WIT Trans Ecol Environ, 215, 357-366. 10.2495/EID180321 